\title{
Design, Characterization and Evaluation of Metallic Nano Biocomposites of Neomycin
}

\author{
Vottikuti Swathi ${ }^{1}$, Maravajhala Vidyavathi ${ }^{1,{ }^{*}}$, T.N.V.K.V. Prasad ${ }^{2, *}$ and R.V. Suresh Kumar ${ }^{3}$ \\ ${ }^{1}$ Institute of Pharmaceutical Technology, Sri Padmavathi Mahila Visvavidyalayam, Tirupati, A.P., India \\ ${ }^{2}$ Department of Soil Science, S.V. Agricultural College Acharya N.G. Ranga Agricultural University, \\ Tirupati-517 502, A.P., India \\ ${ }^{3}$ Department of Surgery and Radiology, SV. Veterinary University, Tirupati, A.P., India
}

\begin{abstract}
Neomycin is formulated into nanoparticles in order to increase the therapeutic efficacy, decrease the dose of drug and to decrease the topical dose related toxic effects. The present study was aimed at the preparation of zinc nanoparticles $(\mathrm{ZN})$, chitosan nanoparticles $(\mathrm{CN})$, zinc neomycin nanoparticles (ZNN) and zinc chitosan neomycin nanoparticles (ZCNN) in order to compare their antibacterial activity. Nanoparticles were prepared by subjecting the nano suspension containing the specified ingredients to stirring at $40^{\circ} \mathrm{C}$ for $4-5 \mathrm{~h}$. The prepared nanoparticles were evaluated for particle size and surface morphology by Transmission Electron Microscopy (TEM), mean particle size and particle size distribution by DLS, percentage yield, loading efficiency, in vitro drug release by diffusion technique and agar cup plate method. TEM microphotographs and zeta sizer analysis revealed that the prepared nanoparticles were in the nanometric range, the particle size and particle size range of ZCNN was less compared to ZNN indicated more surface area of ZCNN. Among all the nanoparticles prepared, percentage yield, loading efficiency, in vitro drug release and zone of inhibition was found to be more for ZCNN. Thus, the results suggested that ZCNN act as promising drug delivery systems with better in vitro characteristics compared to other nanoparticles with increased therapeutic activity of neomycin.
\end{abstract}

Keywords: Chitosan, Nanoparticles, Neomycin, Zinc, Zinc neomycin.

\section{INTRODUCTION}

Nanotechnology deals with design, characterization and application of various structures, devices and systems by controlling the size and shape at nanometric scale. Nanoparticles can be defined as sub-micron sized colloidal particles composed of synthetic or semi-synthetic polymers having size range of $1 \mathrm{~nm}$ to $1000 \mathrm{~nm}$ [1]. They contain macromolecular materials in which the active principle (drug / biologically active agent) is dissolved or entrapped or encapsulated or adsorbed or attached [2]. They act as carrier for various chemotherapeutic agents and biomolecules such as proteins, drugs, vaccines and genes. Nanoparticles can be easily up taken by the cells and can reach every cell to produce effective delivery when compared with conventional carrier [3]. These nanoparticles when coated with polymers provide an increased therapeutic benefit by minimizing the side effects [4]. These enable the drug to enhance the bioavailability, decrease the toxicity [5], decreases the frequency of administration, drug targeting [6].

Metallic nanoparticles are sub-nanosized colloidal particles produced from metals are used as biosensors,

*Address correspondence to these authors at the Institute of Pharmaceutical Technology, Sri Padmavathi Mahila Visvavidyalayam, Tirupati, A.P., India; Tel: +91-9949576350; E-mail: vidyasur@rediffmail.com

Institute of Frontier Technology, Regional Agricultural Research Station, Acharya N G Ranga Agricultural University, Tirupati-571 502., A.P., India; E-mail: tnvkvprasad@gmail.com catalysts and targeted drug delivery systems. Advantages of metallic nanoparticles include high surface to volume ratio [7, 8], uniform size distribution, better optical properties $[9,10]$, better interaction with the biomolecules both at the surface and interior of the cell. Zinc oxide nanoparticles have anti-bacterial, antifungal and growth promoting activity. Several antimicrobial mechanisms of zinc oxide were supposed as (i) hydrogen peroxide, which is generated from the surface of zinc oxide, can penetrate through the cell membrane, produce some type of injury, and inhibit the growth of the cells [11] (ii) the affinity between zinc oxide and bacterial cells is an important factor for antibacterial activity.

Chitosan is used as a polymer because it exhibits antimicrobial activity against bacteria [12], fungi and yeast, biocompatible [13], non-toxic, biodegradable [14, $15]$, used as wound healing accelerator $[16,17]$ as it enhances the function of polymorph nuclear cells, macrophages and enhances fibroblastic proliferation of migration [18]. These properties render chitosan a very attractive material as a drug delivery carrier [19-21]; hence chitosan nanoparticles have been extensively developed and explored for pharmaceutial action in past two decades.

Nanoparticles have a relatively large (functional) surface which is able to bind, adsorb and carry other 
compounds such as drugs, probes and proteins. Because of their ability to carry the drugs and proteins, zinc chitosan neomycin nanoparticles were formulated to enhance the therapeutic efficacy of neomycin to decrease the dose related toxic effects of neomycin ointment such as hypersensitivity reactions, rashes and burning sensation by decreasing the dose of the drug and enhancing its effect. Zinc and chitosan were used because of their antibacterial activity [22] and in order to determine the individual contribution for antibacterial activity in zinc chitosan neomycin nanoparticles.

\section{MATERIALS AND METHODS}

\section{Materials}

Neomycin (Gift sample from Natco pharma Pvt limited, Hyderabad), chitosan (Sigma Aldrich, Hyderabad), agar, beef extract, (Himedia Pvt.limited, Mumbai), methanol, Polyethylene glycol (PEG) 400 (M.wt.420) and PEG 4000 (M.wt.3000), acetic acid (Sdfine, Mumbai), Bacillus subtilis, Staphylococcus aureus \& Escherichia coli, Pseudomonas aeruginosa obtained from NCL, Pune. All the chemicals and reagents used were of analytical and pharmaceutical grade.

\section{Methods}

\section{Preparation of Zinc Chitosan Neomycin Nanoparticles}

$2 \%$ neomycin, $2 \%$ chitosan and $0.2 \%$ nano zinc oxide solutions were prepared. $50 \mathrm{ml}$ of zinc oxide solution was added to $40 \mathrm{ml}$ each of neomycin and chitosan solutions. The preparation of nanoscale zinc oxide was done using oxalate decomposition method. The above solution was stirred continuously using magnetic stirrer by heating at $40^{\circ} \mathrm{C}$ for $4-5 \mathrm{hr}$ followed by centrifugation. The sediment was dried and the dried nanoparticles were evaluated. Different following nanoparticles (Table 1) were prepared by the same procedure.

\section{Characterization of Nanoparticles}

\section{A. Particle Size and Surface Morphology \\ i. TEM Analysis}

The morphological characteristics of nanoparticles were determined by Transmission electronic microscopic (TEM) TEM1200EXJEOL, Japan. Specified quantity of ZNN and ZCNN were placed on the carbon coated copper grid making a thin film of sample on the grid and extra sample was removed using the cone of a blotting paper \& kept in grid box sequentially. Then TEM microphotographs of nanoparticles (ZNN and ZCNN) were taken

\section{ii. Particle Size Measurements}

The particle size and size distribution of the drug loaded nanoparticles (ZNN and ZCNN) were measured using Dynamic Light Scattering technique (DLS) (Nanopartica SZ 100, Horiba, Singapore) at 170 degree scattering angle. The zeta potential was also measured using the same instrument.

\section{B. Compatibility Studies}

\section{a. UV-Vis Spectroscopy}

The compatibility of drug and nanoparticles was studied by using Uv-Vis spectrophotometer (Shimadzu) in scan mode.

\section{b. FT-IR Spectroscopy}

This study was carried using Tensor 27 (Bruker) to find out the compatibility between drug (neomycin), polymer (chitosan) and zinc by scanning from $4000 \mathrm{~cm}$ ${ }^{1}$ to $400 \mathrm{~cm}^{-1}$ in FT-IR spectrophotometer. Samples were prepared for drug neomycin, polymer chitosan, and the nanoparticle of drug, polymer and zinc. The possible interaction between neomycin, chitosan and zinc was accessed by comparing FTIR spectra of pure drug (neomycin), polymer (chitosan) and nanoparticle formulation.

Table 1: Composition of Various Nanoparticles Formulated

\begin{tabular}{|c|c|c|c|c|}
\hline S. No. & Code of the formulation & Neomycin $\%$ & Chitosan $\%$ & Nano zinc oxide \% \\
\hline \hline 1. & ZN (NP1) & - & - & $0.2 \%$ \\
\hline 2. & CN (NP2) & - & $2 \%$ & $0.2 \%$ \\
\hline 3. & ZNN (NP3) & $2 \%$ & - & $0.2 \%$ \\
\hline 4. & ZCNN (NP4) & $2 \%$ & $2 \%$ & \\
\hline
\end{tabular}




\section{Percentage Yield}

The formulation is centrifuged and sediments were dried. Then percentage yield was calculated as follows:

$\%$ Yield $=\frac{\text { Nanoparticles weight } \mathrm{X} 100}{\text { Total solids weight }}$

Total solids weight $=$ weight of nano zinc oxide + weight of neomycin + weight of chitosan.

\section{Loading Efficiency}

Nanosuspension with known amount of drug was centrifuged at $5000 \mathrm{rpm}$ for $15 \mathrm{~min}$. The supernatant solution was separated. $5 \mathrm{ml}$ of supernatant was mixed with $100 \mathrm{ml}$ of distilled water. Absorbance was measured using UV Spectrophotometer using distilled water as blank. The amount of drug present in the supernatant was calculated from which the amount of drug entrapped and \% entrapment was determined.

Loading efficiency $=\frac{\text { Total amount of drug }- \text { Amount of unbound drug }}{\text { Nanoparticles weight }} \times 100$

\section{E. In Vitro Drug Release Studies}

\section{i. In Vitro Drug Release by Diffusion Studies}

In vitro drug release studies were conducted to estimate the rate of release of drug and to find the time taken to release the total drug [23]. The diffusion studies were carried out using dialysis membrane. $5 \mathrm{ml}$ of formulation was accurately placed in this assembly. The cylinder was suspended in $50 \mathrm{ml}$ of dissolution medium maintained at $37 \pm 5^{\circ} \mathrm{C}$. So that the membrane just touched the receptor medium surface. The dissolution medium was stirred at lower speed using magnetic stirrer. Aliquots of samples were withdrawn at regular intervals and replaced with equal volume. The samples were analyzed by UV-Visible spectrophotometer and cumulative percentage release of formulations was specified in the tabular form. The quantity of drug equivalent to $10 \mathrm{mg}$ was taken for diffusion study.

\section{ii. In Vitro Antibacterial Activity by Agar Cup Plate Method}

The prepared nanoparticles were evaluated for antibacterial activity against four different strains with agar cup plate method by measuring the zone of inhibition of microorganisms using gram positive and gram negative organisms. The zone of inhibition was determined for ZN, CN, ZNN and ZCNN formulations separately by incubating for $24 \mathrm{hr}$ at $37 \pm 2^{\circ} \mathrm{C}$. Zone of inhibition was determined using antibiotic zone reader. This was done in triplicate and average diameter was noted.

\section{RESULTS}

\section{A. Particle Size and Surface Morphology}

\section{i. TEM Analysis}

Particle size and surface morphology of ZNN and ZCNN were determined by Transmission Electron Microscopy and its TEM microphotographs are shown in Figures 1 and 2 respectively. The particle size of ZNN was found to be in the range of $32-130 \mathrm{~nm}$ and ZCNN was found to be in the range of $34-120 \mathrm{~nm}$.

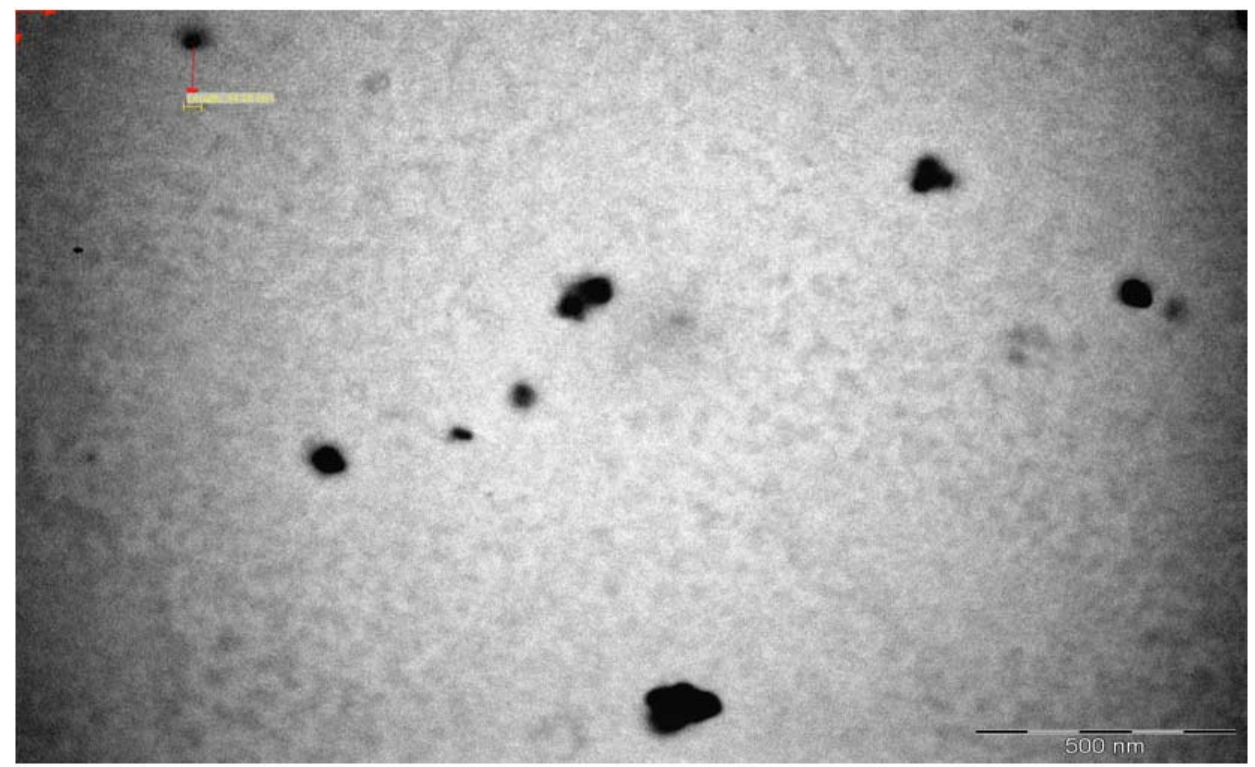

Figure 1: TEM microphotographs of zinc neomycin nanoparticles (ZNN). 


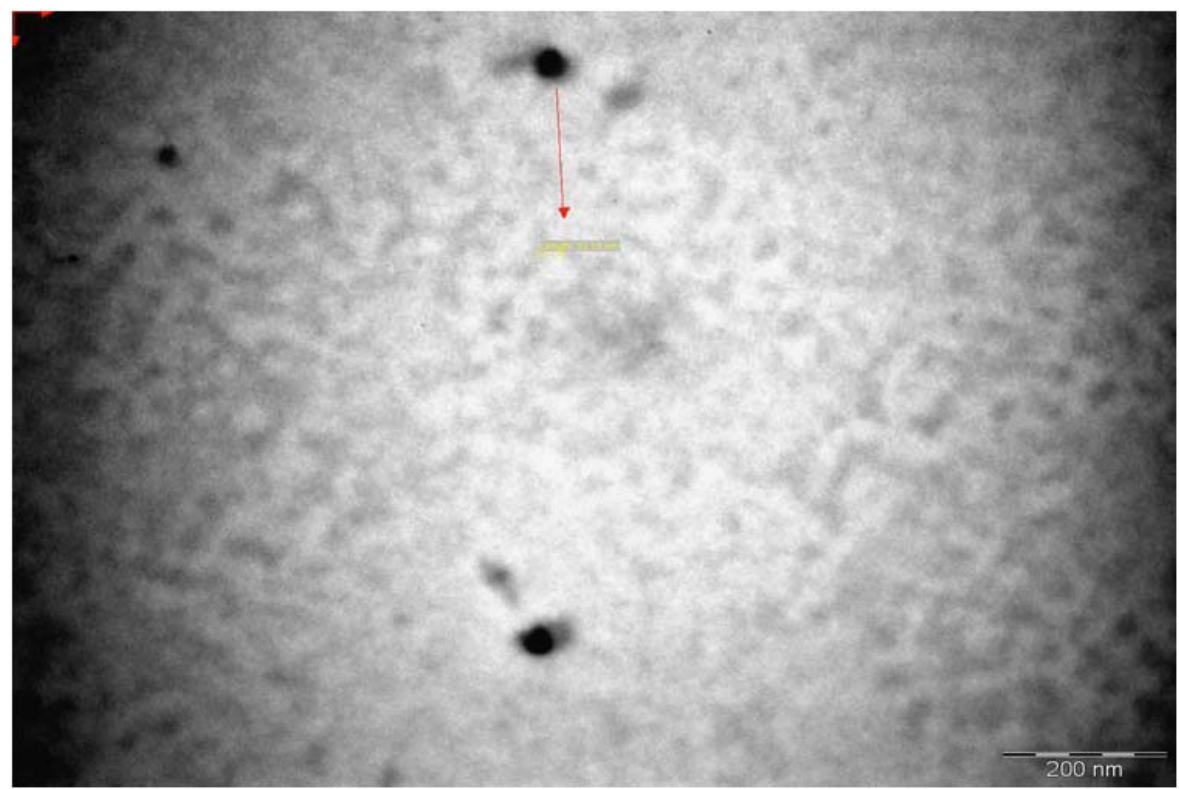

Figure 2: TEM microphotographs of zinc chitosan neomycin nanoparticles (ZCNN).

\section{ii. Particle Size Determination-Dynamic Light Scattering Technique (DLS)}

Mean particle size and particle size distribution of ZNN and ZCNN were determined by using DLS technique. The hydrodynamic radius and size distribution of ZNN and ZCNN were shown in Figures $\mathbf{3}$ and 4 respectively. The decrease in size of nanoparticle by incorporation of polymer was also observed.

\section{B. Compatibility Studies}

The compatibility of neomycin with chitosan and zinc in zinc chitosan neomycin nanoparticles was

Calculation Results

\begin{tabular}{|c|c|c|c|c|}
\hline Peak No. & S.P.Area Ratio & Mean & S. D. & Mode \\
\hline 1 & 1.00 & $129.5 \mathrm{~nm}$ & $6.6 \mathrm{~nm}$ & $128.2 \mathrm{~nm}$ \\
\hline 2 & $\ldots$ & $\cdots \mathrm{nm}$ & $\cdots \mathrm{nm}$ & $\cdots \mathrm{nm}$ \\
\hline 3 & $\ldots$ & $\cdots \mathrm{nm}$ & $\cdots \mathrm{nm}$ & $\ldots \mathrm{nm}$ \\
\hline Total & 1.00 & $129.5 \mathrm{~nm}$ & $6.6 \mathrm{~nm}$ & $128.2 \mathrm{~nm}$
\end{tabular}

\section{Histogram Operations}

Size (Median)

$\%$ Cumulative (2)

$\%$ Cumulative (6)

$\%$ Cumulative (10)
: $128.2 \mathrm{~nm}$

: $10.0(\%) \cdot 120.6(\mathrm{~nm})$

: $50.0(\%)-128.2(\mathrm{~nm})$

: $90.0(\%)-142.6(\mathrm{~nm})$

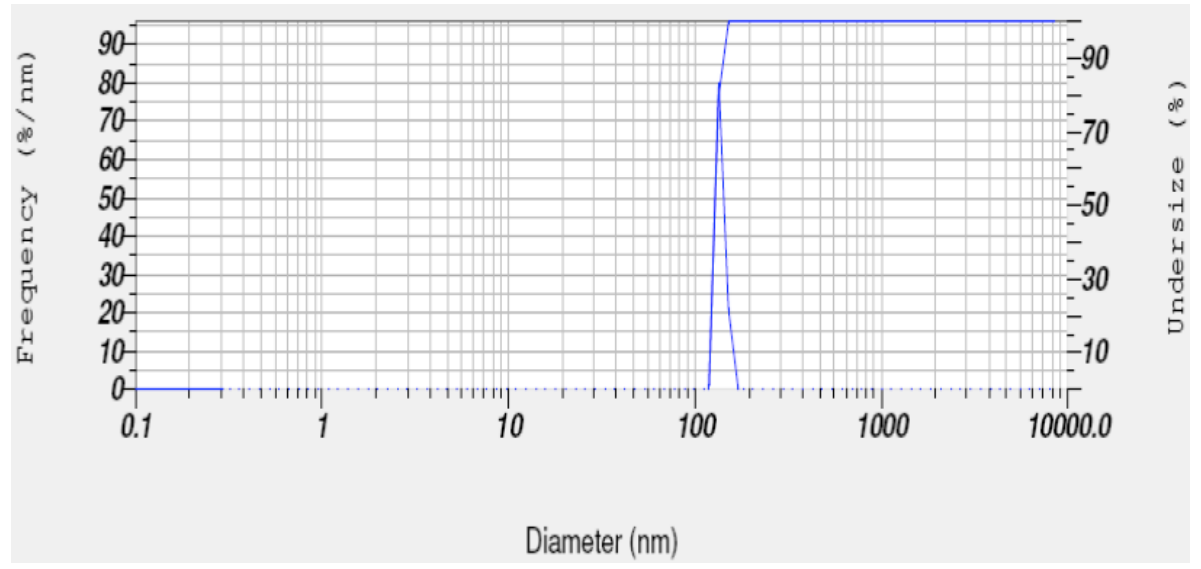

Figure 3: Particle size determination of zinc neomycin nanoparticles (ZNN). 
Calculation Results

\begin{tabular}{|c|c|c|c|c|}
\hline Peak No. & S.P.Area Ratio & Mean & S. D. & Mode \\
\hline 1 & 1.00 & $111.5 \mathrm{~nm}$ & $1.6 \mathrm{~nm}$ & $111.6 \mathrm{~nm}$ \\
\hline 2 & $\cdots$ & $\cdots \mathrm{nm}$ & $\cdots \mathrm{nm}$ & $\cdots \mathrm{nm}$ \\
\hline 3 & $\cdots$ & $\cdots \mathrm{nm}$ & $\cdots \mathrm{nm}$ & $\cdots \mathrm{nm}$ \\
\hline Total & 1.00 & $111.5 \mathrm{~nm}$ & $1.6 \mathrm{~nm}$ & $111.6 \mathrm{~nm}$
\end{tabular}

Histogram Operations

Size (Median)

$\%$ Cumulative (2)

$\%$ Cumulative (6)

$\%$ Cumulative (10)

: $111.6 \mathrm{~nm}$

: $10.0(\%)-106.2(\mathrm{~nm})$

: $50.0(\%)-111.6(\mathrm{~nm})$

: $90.0(\%)-117.3(\mathrm{~nm})$

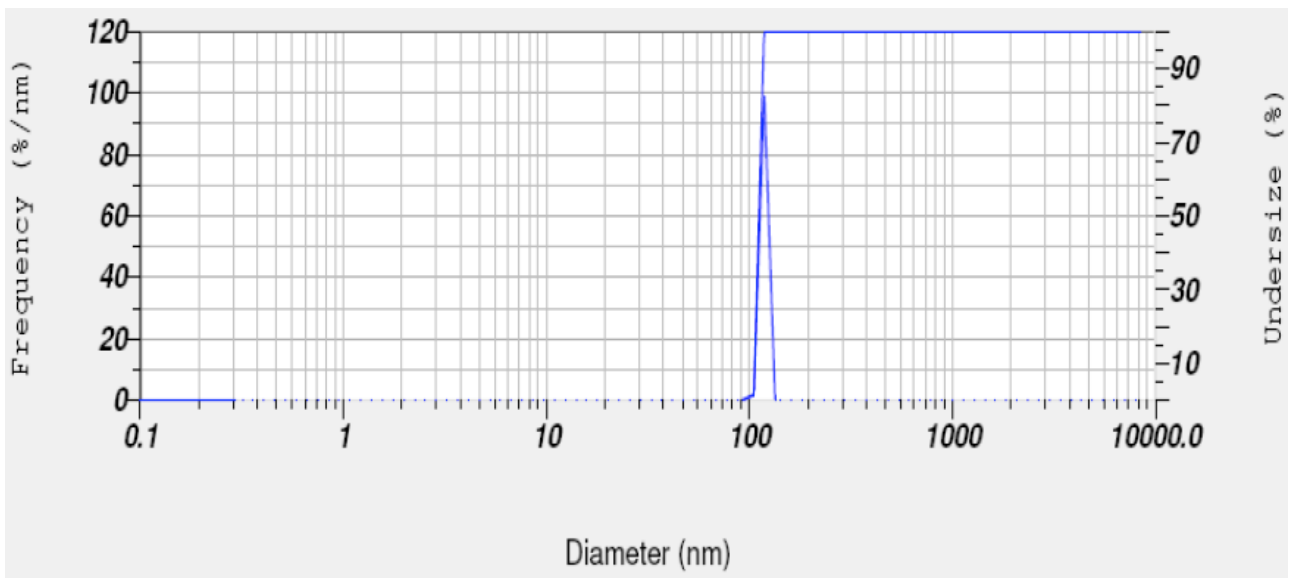

Figure 4: Particle size determination of zinc chitosan neomycin nanoparticles (ZCNN).

determined through UV scan, FT-IR analysis. The UV spectrum of pure drug solution and zinc chitosan neomycin nanoparticle formulation was identical and the characteristic absorption maximum was appeared at $273 \mathrm{~nm}$. The FT-IR spectra of pure neomycin, pure chitosan and ZCNN were obtained and the characteristic bands of pure neomycin were almost found in FT-IR spectrum of ZCNN similar to pure neomycin spectrum.

\section{Percentage Yield \& Loading Efficiency}

The \% yield of nanoparticles was varied from 72.45 \pm 0.12 to $83.04 \pm 0.31$ as given in Table 1 . The loading efficiency of ZNN and ZCNN was found to be $75.92 \% \pm$ 0.23 and $80.11 \% \pm 0.32$ respectively as given in Table 2.

Table 2: Percentage Yield and Loading Efficiency of Various Nanoparticles

\begin{tabular}{|c|c|c|c|}
\hline SI. No. & Formulation code & $\begin{array}{c}\text { \% Yield } \\
\text { (Mean } \pm \text { S.D) }\end{array}$ & $\begin{array}{c}\text { Loading efficiency } \\
\text { (\%) (Mean } \pm \text { S.D) }\end{array}$ \\
\hline \hline 1. & NP1 & $72.45 \pm 0.12$ & - \\
\hline 2. & NP2 & $74.94 \pm 0.13$ & - \\
\hline 3. & NP3 & $75.13 \pm 0.26$ & $75.92 \pm 0.23$ \\
\hline 4. & NP4 & $79.13 \pm 0.26$ & $80.11 \pm 0.32$ \\
\hline
\end{tabular}

\section{In Vitro Drug Release}

\section{i. By Diffusion Studies}

The invitro drug release rate from zinc neomycin nanoparticles (NP3) and zinc chitosan neomycin nanoparticles (NP4) is shown in Figure 5.

\section{ii. By Agar Cup Plate Technique}

The antibacterial activity of different nanoparticles was determined against four different bacteria and shown in Figure 6.

\section{DISCUSSION}

In the present study, ZNN and ZCNN were prepared and characterized to find out the effect of zinc and chitosan on antibacterial activity of ZNN. It was also 


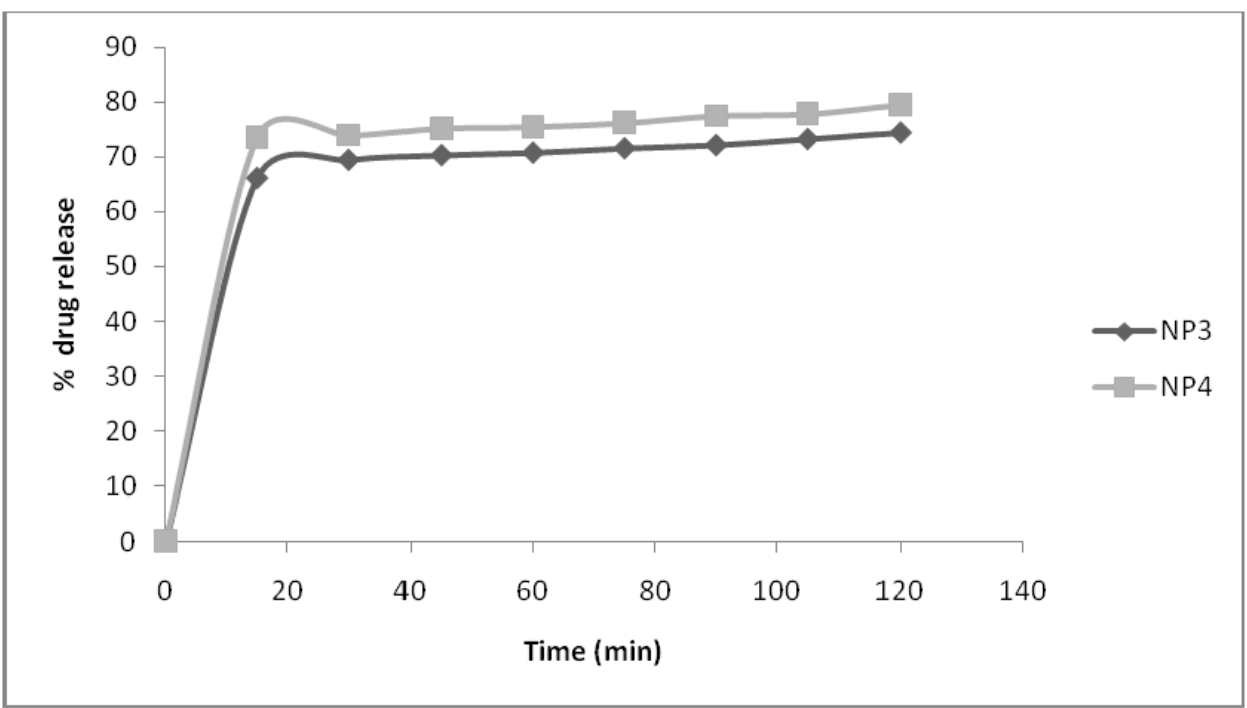

Figure 5: \% Drug release Vs Time profile of zinc neomycin nanoparticles (ZNN) and zinc chitosan neomycin nanoparticles (ZCNN).

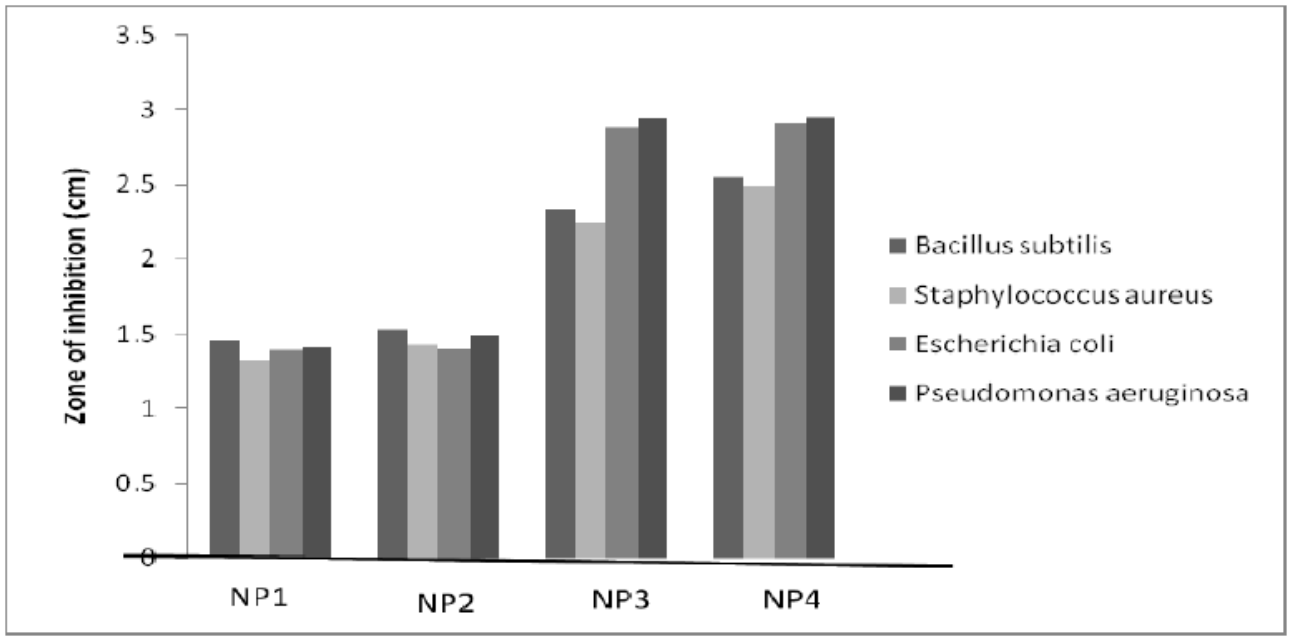

Figure 6: Zone of inhibition of Zinc nanoparticles, Chitosan nanoparticles, Zinc neomycin nanoparticles and Zinc chitosan neomycin nanoparticles (NP1,NP2, NP3, NP4 respectively).

compared with pure zinc nanoparticles $(\mathrm{ZN})$ and pure chitosan nanoparticles $(\mathrm{CN})$. These zinc chitosan neomycin nanoparticles were formulated to get maximum loading efficiency, invitro drug release by diffusion studies and agar cup plate method.

It is evident from the TEM microphotographs (Figures $\mathbf{1}$ and 2) that ZCNN are more spherical in shape with smooth surface when compared to ZNN. The particle size of ZNN and ZCNN was found to be $128 \mathrm{~nm}$ and $111.6 \mathrm{~nm}$ respectively. A significant decrease $(P<0.05)$ in particle size was found in case of ZCNN when compared to ZNN, indicated the incorporation of chitosan into the structure of nanoparticles lead to significant decrease in the size of nanoparticles.
The decrease in size of nanoparticle by incorporation of polymer was also confirmed by particle size analysis using Nanopartica SZ-100. The mean hydrodynamic radius of ZNN and ZCNN was found to be $128 \mathrm{~nm}$ and $111.6 \mathrm{~nm}$ as shown in Figures 3 and 4 . As the nanoparticles made with chitosan (ZCNN) are more reticulated, it leads to smaller size when compared to nanoparticles prepared without polymer $(Z N N)$. The particle size, mean particle size was significantly decreased in ZCNN when compared to ZNN. This reticulation of nanoparticle by the polymer lead to the formation of more interlacing links to a closed network which finally lead to decreased particle size, so it also allowed to capture more drug which was observed with increased loading efficiency by addition of polymer. The narrow size distribution or more 


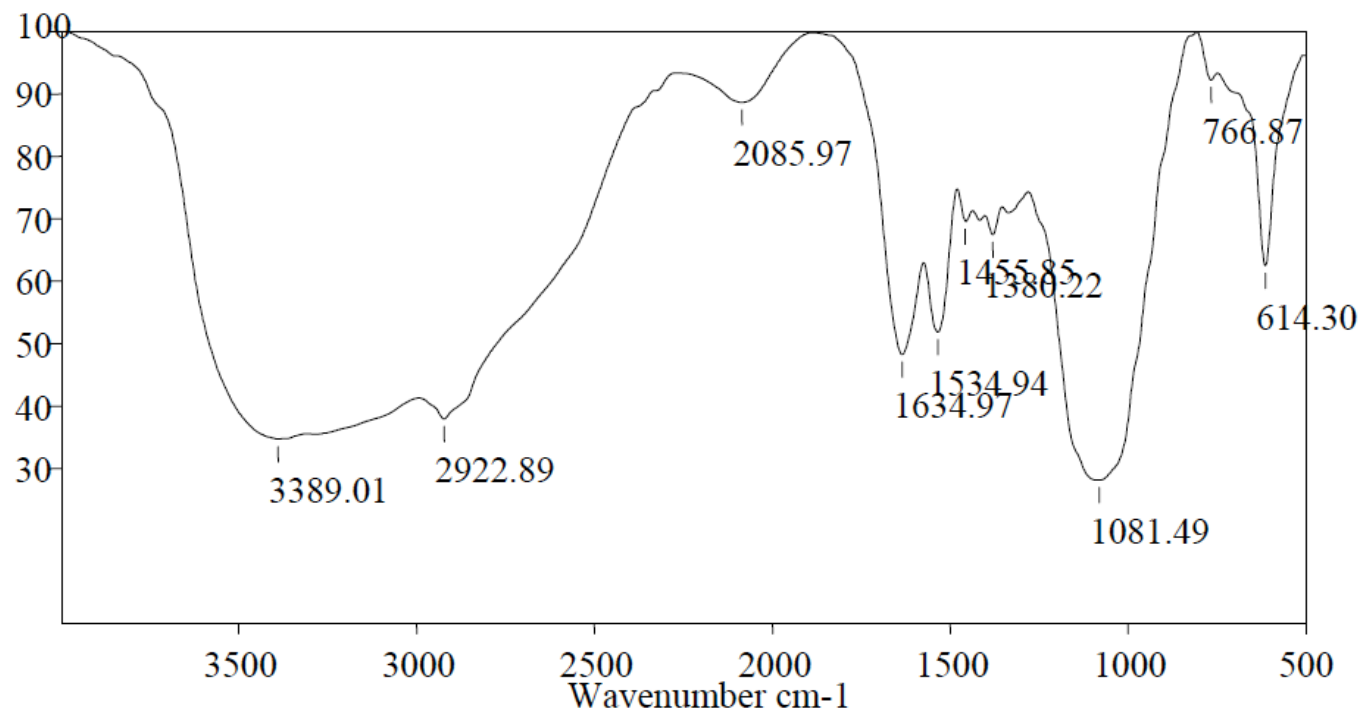

Figure 7: FT-IR Spectrum of zinc chitosan neomycin nanoparticles (ZCNN).

uniform size distribution was found with ZCNN than ZNN, may be due to reticulation effect of polymer.

The compatibility of neomycin with chitosan and zinc in zinc chitosan neomycin nanoparticles was determined through UV-Vis spectroscopy and FTIR analysis. The UV spectrum of pure drug solution and zinc chitosan neomycin nanoparticle formulation was identical and the characteristic absorption maximum was appeared at $273 \mathrm{~nm}$.

FTIR spectrum of pure neomycin demonstrated the characteristic absorption peaks at $3240 \mathrm{~cm}^{-1}$ for $\mathrm{O}-\mathrm{H}$ stretching conjugated with $\mathrm{N}-\mathrm{H}$ stretching and aromatic $\mathrm{C}-\mathrm{H}$ stretching, at $1341 \mathrm{~cm}^{-1}$ for C-N stretching, at 1075 $\mathrm{cm}^{-1}$ for C-O stretching, at $1530 \mathrm{~cm}^{-1}$ for $-\mathrm{C}-\mathrm{C}$ stretching. The absorption peaks with zinc chitosan neomycin nanoparticles were almost similar to those obtained with the pure drug and polymer (Figure 7). Further the peaks at $3395,2922,1261,1073 \mathrm{~cm}^{-1}$ in FTIR spectrum of pure chitosan indicated the presence of $\mathrm{O}-\mathrm{H}$ stretching, $\mathrm{C}-\mathrm{H}$ stretching, C-O stretching and $\mathrm{C}-\mathrm{O}$ ether stretching in chitosan structure. These peaks are also found in spectrum of formulation indicated the incorporation of chitosan in the formulation and compatibility of drug with both zinc and polymer.

The \% yield of nanoparticles varied from $72.45 \pm$ 0.12 to $79.13 \pm 0.26$. Among all the prepared nanoparticles the \% yield was found to be less for zinc nanoparticles (NP1) i.e., $72.45 \%$ and highest for zinc chitosan neomycin nanoparticles (ZCNN) i.e., 79.13\% as shown in Table 2 . The \% yield was found to be increased with incorporation of chitosan into zinc neomycin nanoparticles (ZNN).
The loading efficiency of ZNN was $75.92 \%$ whereas $80.11 \%$ in case of ZCNN (Table 2). The loading efficiency ZCNN was increased when compared to ZNN. This indicated an increase in loading efficiency with incorporation of polymer, may be due to formation of reticulated polymeric sheath around the nanoparticles and it ultimately increased the drug loading [24-27].

The in vitro \% drug release by diffusion studies was determined for all the nanoparticles for $2 \mathrm{hrs}$. At the end of $2 \mathrm{~h}$ the in vitro \% drug release of different nanoparticles was found to be between 74.67 to 79.56 $\%$ as shown in Figure 5 . The in vitro \% drug release from ZNN was found to be $74.67 \%$ and from ZCNN it was found to be $79.56 \%$. It may be due to the role of polymer on diffusion of drug neomycin. It can be supported by the reports about the capacity of chitosan in promoting macromolecules permeation through wellorganized membrane [28-30].

The in vitro drug release was also determined by measuring the zone of inhibition using agar cup plate technique against four different strains of gram positive and gram negative microorganisms as given in Figure 6. The antibacterial activity of drug loaded nanoparticles was significantly more than blank nanoparticles against all selected species $(P<0.05)$. Among the prepared nanoparticles, zinc nanoparticles were found to have the least antibacterial activity and ZCNN were found to possess highest antibacterial activity (Figure 6). It may be due to the synergistic effect of all the ingredients i.e., zinc, chitosan, neomycin present in the formulation and also due to increased surface area with decrease in particle size. 
Increase in antibacterial effect was found by increased drug loading, when compared with $\mathrm{ZN}$ and $\mathrm{CN}$. However, pure chitosan nanoparticles (CN) have shown higher in vitro antibacterial activity than zinc nanoparticles, indicated more capacity of chitosan to act against different strains of bacteria, ZCNN was found to possess highest anti-bacterial activity when compared to other formulations [31].

\section{CONCLUSION}

A novel nanoparticle based drug delivery system with the slow-releasing action has been demonstrated. Of all the nanoparticle formulations, ZCNN were found to possess maximum percentage yield, loading efficiency, in vitro drug release and in vitro antimicrobial activity. This may be due to smaller particle size and uniform size distribution of ZCNN compared to ZNN, which enhances the surface area. This strongly suggests the use of nanoparticles in drug delivery systems in the form of metallic nanobiocomposites as better drug delivery systems to reduce the dose of the drug, thereby to reduce the dose related toxicities by enhancing the efficacy of the drug.

\section{REFERENCES}

[1] Lang L, Ping J, Ming C, Guoliang Z, Fengbao Z. 5Fluorouracil-loaded self-assembled $\mathrm{pH}$-sensitive nanoparticles as novel drug carrier for treatment of malignant tumors. Chin J Chem Eng 2006; 377-82.

[2] Kumar GA, Bhat A, Rani S. Preparation and characterization of diltiazem nanocapsules: Influence of various polymers. Asian J Pharm 2010; 224-34.

[3] Moudgil BS, Ying JY. Calcium-doped organosilicate nanoparticles nanoparticles as gene delivery vehicles for bone cells. Sci Tech Adv Mater 2007; 3130-35.

[4] Mohanraj VJ, Chen Y. Nanoparticles - A review. Trop J Pharm Re 2006; 561-73.

[5] Mohsen J, Zahra B. Protein nanoparticle: A unique system as drug delivery vehicles. Afr J Biotechnol 2008; 4926-34.

[6] Yokoyama M, Okano T. Advances in drug delivery review, 1996; 77-80. http://dx.doi.org/10.1016/S0169-409X(96)00439-5

[7] Sau TKA, Rogach L, Jackel F, Feldmann J. Properties and applications of colloidal nonspherical noble metal nanoparticles. Adv Mater 2010; 1805-25.

[8] Sperling RA, Gil PR, Zhang F, Parak WJ. Biological applications of gold nanoparticles. Chem Soc Rev 2008; 1896-908.

http://dx.doi.org/10.1039/b712170a

[9] Jain PK, Huang X, El-Sayed IH. Noble metals on the nanoscale: Optical and photothermal properties and some applications in imaging, sensing, biology, and medicine. Acc Chem Res 2008; 1578-86.

[10] Lee KS, El-Sayed MA. Gold and silver nanoparticles in sensing and imaging: sensitivity of plasmon response to size, shape, and metal composition. J Phys Chem B 2006; 1922025.

http://dx.doi.org/10.1021/jp062536y
[11] Meruvu H, Vangalapati M, Chippada SC, Bammidi SR. Synthesis and characterization of zinc oxide nanoparticles and its antimicrobial activity against Bacillus subtilis and Escherichia coli. Rasayan J Chem 217-222.

[12] Wang C, Liu L-L, Zhang A-T, Xie P, Lu J-J, Zou X-T. Antibacterial effects of zinc oxide nanoparticles on Escherichia coli K88. Afr J Biotechnol 2012; 10248-54.

[13] Mohy Eldin MS, Soliman EA, Hashem AI, Tamer TM. Chitosan modified membranes for wound dressing applications: Preparations, characterization and bioevaluation. Trends Biomater Artif Organs 2008; 154-64.

[14] Shelma R, Paul W, Sharma CF. Chitin nanosphere reinforced thin chitosan films for wound healing application. Trends Biomater Artif Organs 2008; 107-11.

[15] Saraswathy G, Pal S, Rose C, Sastry TP. A Novel Bioinorganic bone implant containing Deglued bone, Chitosan and Gelatin. B Mater Sci 2001; 415-20.

[16] Emir BD, Raphael MO. Persectives on: Chitosan Drug Delivery Systems Based on their Geometries. J Bioact Compat Polym 2006; 351-68.

[17] Daniela E, Camelia EO. Functionalized Chitosan and its use in Pharmaceutical, Biomedical and Biotechnological Research. Chem Eng Commun 2008; 1269-91.

[18] Moudgil BS, Ying JY. Calcium-doped organosilicate nanoparticles as gene delivery vehicles for bone cells. Sci Tech Adv Mater 2007; 3130-35.

[19] Su CH, Sun CS, Juan SW, Hu CH, Ke WT, Sheu MT. Funga mycelia as the source of chitin and polysaccharides and their application as skin substitutes. Biomaterials 1997; 1169-74.

[20] Muzzarelli RAA, Sipos L. Chitosan for the collection from seawater of naturally occurring zinc, cadmium, lead and copper. Talanta 1971; 853-58.

[21] Pandey R, Ahmad Z, Sharma S, Khullar GK. Nanoencapsulation of azole antifungals, Potentia applications to improve oral drug delivery. Int J Pharm 2005; 1169-74.

[22] Gupta H, Aqil M, Khar RK, Ali A, Bhatnagar A, Mittal G Sparfloxacin-loaded PLGA nanoparticles for sustained ocular drug delivery. Nanomedicine NBM 2010; 6: 324-33. http://dx.doi.org/10.1016/i.nano.2009.10.004

[23] Tamizhrasi S, Shukla A, Shivkumar T, Rathi V, Rathi JC. Formulation and evaluation of lamivudine loaded polymethacrylic acid nanoparticles. Int J Pharm Tech Res 2009; 411-15

[24] Hoa LTM, Chi NT, Triet NM, Nhan LNT. Preparation of drug nanoparticles by emulsion evaporation method. J Phys Con Ser 2009; 1-4.

[25] Hou Z, Wei H, Wang Q, Sun Q. New method to prepare mitomycin C loaded PLA-nanoparticles with high drug entrapement efficiency. Nanoscale Res Let 2009.

[26] Kumar PV, Jain NK. Suppression of agglomeration of ciprofloxacin-loaded human serum albumin nanoparticles. The AAPS PharmSciTech 2007; 8(1): E1-E6. http://dx.doi.org/10.1208/pt0801017

[27] Wilson B, Samanta MK, Santhi K, Kumar KPS Paramakrishnan N, Suresh B. Targeted delivery of tacrine into the brain with polysorbate 80-coated poly(n-butyl cyanoacrylate) nanoparticles. Eur J Pharm Biopharm 2008; 75-84. http://dx.doi.org/10.1016/i.ejpb.2008.03.009

[28] Pandey R, Ahmad Z, Sharma S, Khullar GK Nanoencapsulation of azole antifungals, Potential applications to improve oral drug delivery Int J Pharm Sci 2005; 268-76.

[29] Arturson P, Lindmark T. Effect of chitosan on permeability of monolayers of intestinal epithelial cells (Caco-2). Pharmacal Res 11, 1358-61. 
[30] Borchard G, Luben HL, De Boer. The potential of mucoadhesive polymers in enhancing intestinal peptide drug absorption: Effects of chitosan glutamate and carbomer on epithelial tight junctions in vitro. J Control Release 1996; 13138.
[31]

Abdelhady MM. Preparation and characterization of chitosan/zinc Oxide nanoparticles for imparting antimicrobial and UV protection to cotton fabric. Int J Car Chem 2012; 612.

http://dx.doi.org/10.1155/2012/840591 